\title{
Coronavirus in the elderly: a late lockdown UK cohort
}

\author{
Authors: Andrew D Kerr ${ }^{A}$ and Sybil RL Stacpoole ${ }^{B}$
}

\section{Objective}

To identify the source of ongoing coronavirus disease 2019 (COVID-19) infections after 4 weeks of lockdown and to characterise the presentation of COVID-19 in the elderly, who represent the highest risk group.

Design

Retrospective observational cohort study of 115 patients at one acute district general hospital with a catchment population of approximately 500,000 people, during weeks 5 and 6 of the UK lockdown.

\section{Results}

More than 2 in 3 of the overall cohort had had contacts with the health and social care system prior to diagnosis. This figure rose to $85 \%$ in those 70 years and over. In the older cohort, the most common reasons for presentation were shortness of breath or falls, and 1 in 3 had neither cough nor fever.

\section{Conclusion}

COVID-19 can present differently in the elderly, overlapping with many common presentations, so focusing testing on those with a cough or fever will miss at least 1 in 3 cases in those over the age of 70. A high degree of vigilance, suspicion and repeated testing is required if streaming into high and low risk areas is to succeed, allowing safe restarting of services such as elective surgery and cancer care.

KEYWORDS: COVID-19, elderly, SARS-CoV-2

DOI: $10.7861 /$ clinmed.2020-0423

\section{Introduction}

The coronavirus disease 2019 (COVID-19) pandemic continues to pose more questions than answers. Why do curves showing numbers of new infections fall relatively rapidly but then plateau, rather than falling back to zero, and where do cases come from after 4 weeks of lockdown? Are presentations the same across the ages, especially in a disease that has a particularly serious effect in the elderly?

While we know that older people with COVID-19 are more likely to be severely affected, admitted to hospital and die, little

Authors: ${ }^{A}$ IMT1, North West Anglia NHS Foundation Trust; ${ }^{B}$ Consultant neurologist, North West Anglia NHS Foundation Trust

\section{What is already known?}

Much speculation has centred on the topic of where COVID-19 cases come from late into lockdown, but little is published to inform this debate. Similarly, while several large patient cohorts have been reported by various countries around the world, little attention has been paid to whether the presentation of COVID-19 may vary in the elderly, who represent the group most likely to be seriously affected.

What is the question?

We therefore sought to assess the epidemiological question of where cases continue to come from in weeks 5 and 6 of lockdown, and the diagnostic question of whether presentations of COVID-19 differ in the elderly.

What was found, and implication for practice now?

The majority of cases of COVID-19 late in lockdown are epidemiologically linked to contact with health and care services, including carers visiting people at home, which is an important public health consideration for ongoing pandemic planning. COVID-19 can present differently in the elderly, overlapping with many common medical presentations, so focusing testing on those with a cough or fever will miss at least 1 in 3 cases in those over the age of 70 .

attention has been paid to how the virus may present specifically in this population. It is critical to understand this, both to optimise identification of disease and treatment in this vulnerable group, but also to allow health care systems to identify patients who are likely to be infectious at the front door and carry out streaming into high-risk and low-risk areas (often termed red and green zones, respectively). This is further complicated by issues with the accuracy of the reverse transcriptase polymerase chain reaction (RT-PCR) laboratory test, and the pre-test probability, which is based upon what we know about the clinical presentation of this new disease. ${ }^{2}$

Initial cohorts were dominated by younger patients, with a progressive aging of cohorts, as seen in Wuhan (median age 49 years), New York (62 years) and Lombardy (67 years)..$^{3-5}$ This rapidly led to recognition of the typical clinical features of cough, fever and shortness of breath, although the prevalence of fever has been variable (range $25.5-88.7 \%$ ). More recently, this triad has been confirmed in a large UK cohort of over 20,000 hospitalised patients with a median age of $73 .^{6}$

Furthermore, data from the COVID Symptom Study smartphonebased app helpfully identify a range of symptoms totalling 11 
(cough, fever, shortness of breath, loss of smell and taste, fatigue, diarrhoea, delirium, loss of appetite, abdominal pain, chest pain, hoarse voice), but again this is mostly based on data from younger patients, with average age in their $40 \mathrm{~s}$.

We therefore sought to assess the epidemiological question of where cases continue to come from in weeks 5 and 6 of lockdown, and the diagnostic question of whether presentations of COVID-19 differ in the elderly.

\section{Methods}

All adult patients who tested positive for COVID-19 on combined nasopharyngeal and throat swabs at one UK regional hospital in weeks 5 and 6 of lockdown (20 April 2020 to 3 May 2020) were identified. Hospital-wide testing (screening of current inpatients and those being admitted through the emergency department) was introduced over the course of week 6; prior to that, testing (with limited capacity) was based on clinical suspicion. Case identification involved running a report of microbiology test results and reviewing all results to identify those that were positive. Electronic medical records were then manually interrogated to provide a high level of clinical detail, involving NerveCentre (observations), Sunquest
ICE (laboratory results and discharge summaries), Xero (radiology; reports and images themselves were reviewed) and E-track (doctor and nursing notes). Categorical data were compared using the $X^{2}$ test, and continuous variables using the Mann-Whitney $U$ test (twotailed), both in Microsoft Excel and taking significance as $p<0.05$. Research approval was provided by the hospital research governance team

\section{Results}

\section{Characteristics of the cohort}

118 patients were identified. Three obstetric patients (for whom no further clinical information was accessible) were excluded from further analysis. The cohort of 115 was subdivided into those under $70(n=40)$, and those 70 years and over $(n=75)$, reflecting UK Government advice that those aged 70 and over should be shielded. All 115 cases identified had symptoms, signs or findings consistent with COVID-19; no asymptomatic patients were found.

Overall, the cohort had a median age of 77.5 years, representing one of the oldest cohorts to date (Table 1). The cohort aged 70 and over had a median age of 84 years with roughly even

Table 1. Cohort demographics and epidemiology of infection with coronavirus-19 in weeks 5 and 6 of lockdown in the UK

\section{Characteristic}

\section{Baseline demographics}

Median age in years, interquartile range

Male

Epidemiological factors

Previous hospitalisation within 28 days

Admitted from a care home

Carers at own home

Works/household works in healthcare

One of following applies: recent admission/care home/carers/health work

Documented household transmission

Working (non-healthcare)

Community (unidentified source)

\section{Comorbidities}

Hypertension

Type 2 diabetes mellitus

Ischaemic heart disease

Dementia

Chronic obstructive pulmonary disease

Chronic kidney disease

Stroke or TIA

Cancer

Asthma

No comorbidities

\section{Whole cohort}

$(\mathrm{N}=115)$

$77.5,51-86$

$66(57.4)$

$22(19.1)$

$46(40.0)$

$18(15.7)$

$6(5.2)$

$81(70.4)$

3 (2.6)

4 (3.5)

$14(12.2)$

$50(43.5)$

$38(33.0)$

$29(25.2)$

$28(24.3)$

$19(16.5)$

$18(15.7)$

$14(12.2)$

11 (9.6)

7 (6.1)

$8(7.0)$

\section{Under 70}

$(n=40)$

Aged $\geq 70$

$(n=75)$

$57.5,47-61$

84, 78-89

0 (75) $36(48)$

(10.0)

$18(24)$

$38(50.7)$

$16(21.3)^{*}$

$1(1.3)^{*}$

$64(85.3)$

1 (1.3)

$0(0)^{*}$

$10(13.3)^{*}$

$16(35)$

$34(45.3)$

24 (32)

$24(32)^{*}$

$26(34.7) *$

15 (20)

$15(20)$

12 (16)

9 (12)

$4(5.3)$

$1(1.3)^{*}$

Values are numbers (percentages) unless stated otherwise. ${ }^{*} p<0.05$, comparing the $<70$ and $\geq 70$ groups. 
split across the sexes, possibly reflecting the higher overall life expectancy of women. The younger cohort, with median age 57.5 , was dominated by male patients $(75 \%)$, as reported in other studies. ${ }^{2-5,6,9}$

\section{Epidemiology}

Overall, more than two in three had had contacts with the health and social care system, either through living in a care home, having carers attend their own home, an earlier admission to hospital or working as, or living with, a carer, nurse or doctor. This figure rose to $85 \%$ in the shielding population aged 70 years and over (Table 1). One in eight of the younger cohort worked in healthcare. This confirms the much discussed hypothesis of the time - that the excellent adherence of the British public to lockdown rules meant that ongoing cases would arise in areas where essential workers must continue to work, and particularly in health and social care, where contact with cases was likely.

\section{Comorbidities}

Those 70 and over were significantly more likely to have comorbidities (Table 1), particularly ischaemic heart disease and dementia, but the cohort overall had a high rate of comorbidities (93\%), which is no doubt related to the previous finding that the majority lived in a care home or had carers at their own home.

\section{Clinical presentation}

Looking at the cohort in its entirety, and studying the triage notes which usually clearly document the reason for presentation to hospital, it is apparent that this varied widely, making it difficult to identify patients who may or may not have COVID-19 at the front door (Fig 1).

Looking specifically at those in the 70 years and over cohort, with greater depth of clinical information from the clerking in the emergency department and re-exploration of the history later in the medical notes, sometimes with collateral from family members, some variations in clinical presentation become apparent (Table 2). Only 1 in 5 presented with the typical triad of fever, cough and shortness of breath, and one in seven of those $\geq 70$ years old presented with a low temperature of $\leq 35.5^{\circ} \mathrm{C}$. Less than half had a high fever at any point of the admission, but nearly two in three had a low temperature (Fig 2); note that all three representative traces are taken from those that survived. By contrast, drowsiness or confusion were relatively common (39\% had one or the other), as were general decline, malaise and reduced oral intake. Falls were a frequent presentation, sometimes complicated by neck of femur fracture or head injury, of considerable relevance to orthopaedic and neurosurgeons. Six patients presented with falls without cough, fever or shortness of breath, and three with isolated chest pain at the point of admission to hospital.

Gastrointestinal symptoms including vomiting, diarrhoea and abdominal pain were also common in the whole cohort, though significantly more so in those under 70 , potentially leading to incorrect diagnoses of urinary tract infections with the combination of lower abdominal pain and fever. Anaemia and gastrointestinal haemorrhage were also observed.

\section{Laboratory investigations}

C-reactive protein (CRP), lymphocyte and platelets counts showed no overall differences according to age (Fig 3 and Table 3). Five patients were excluded from this analysis due to long-standing haematological disturbances. Previously reported poor prognostic markers include a lymphocyte count of $<0.8 \times 10^{9} / \mathrm{L}$, platelets $<100 \times 10^{9} / \mathrm{L}$, white cell count $>10 \times 10^{9} / \mathrm{L}$ and CRP $>125.9,10$ Our sample size was small to look at laboratory variables, but there were notable findings in that CRP was not significantly different in those who died versus those who survived in the older cohort while a higher platelet count, though still within the normal range, at presentation was a poor prognostic factor in the older cohort. A high white cell count was a bad prognostic sign in both groups.

\section{Radiological investigations}

Around one in three patients with coronavirus-19 had a normal chest X-ray at presentation (Table 4). Radiographic changes, when present, were also often unilateral. Around half of the younger cohort had bilateral changes, as did a third of the older cohort,

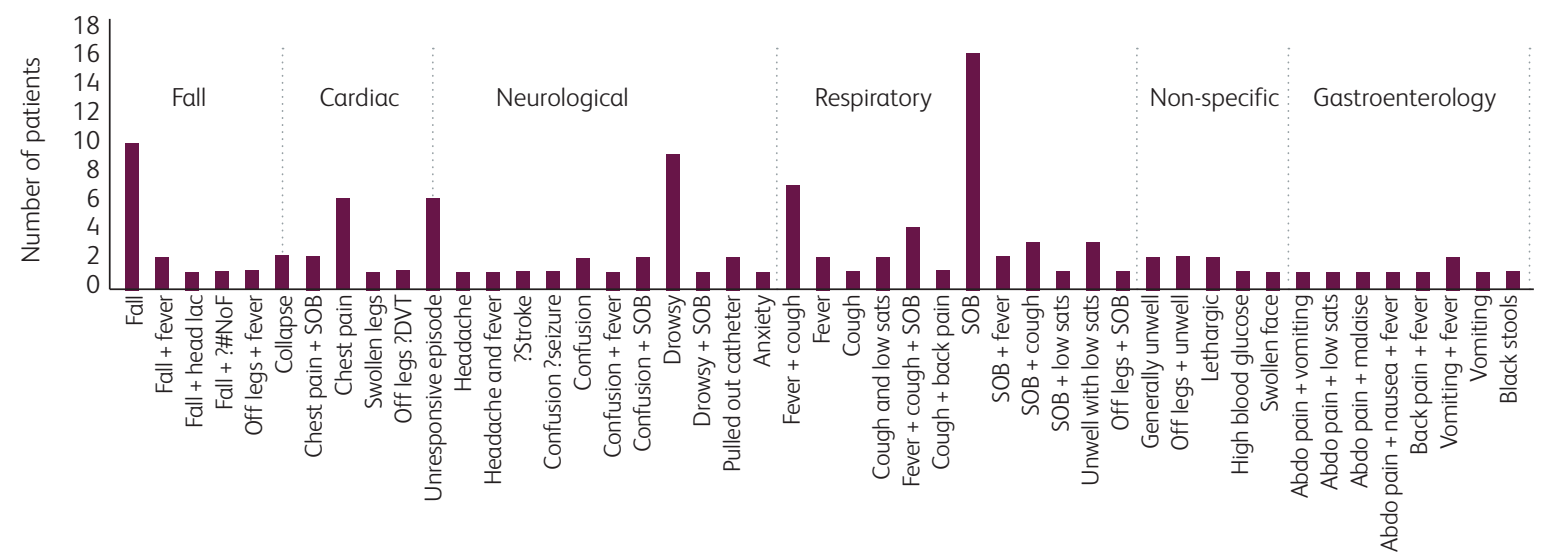

Fig 1. Reason for presentation to hopsital, according to emergency department triage records. Abdo = abdominal; DVT = deep vein thrombosis; lac = laceration; \#NoF = fractured neck of femur; $\mathrm{SOB}=$ shortness of breath. 
Table 2. Presenting symptoms and signs, along with temperature during hospital admission, in patients diagnosed with COVID-19 in weeks 5 and 6 of lockdown in the UK

\begin{tabular}{|c|c|c|c|}
\hline Presenting symptoms & $\begin{array}{l}\text { Whole cohort } \\
(\mathrm{N}=115)\end{array}$ & $\begin{array}{l}\text { Under } 70 \\
(n=40)\end{array}$ & $\begin{array}{l}\text { Aged } \geq 70 \\
(n=75)\end{array}$ \\
\hline Fever (or reported chills) or cough & $82(71.3)$ & $33(82.5)$ & $49(65.3)$ \\
\hline Cough & $59(51.3)$ & $27(67.5)$ & $32(42.7)^{*}$ \\
\hline Fever or reported chills & $66(57.4)$ & $26(65)$ & $40(53.3)$ \\
\hline Shortness of breath & $61(53.0)$ & $24(60)$ & $37(49.3)$ \\
\hline One of cough, fever or shortness of breath & $92(80)$ & $36(90)$ & $56(74.7)$ \\
\hline All of cough, fever and shortness of breath & $32(27.8)$ & $16(40)$ & $16(21.3)^{*}$ \\
\hline Chest pain & $21(18.3)$ & $10(25)$ & $11(14.7)$ \\
\hline General malaise/off food & $32(27.8)$ & $11(27.5)$ & $21(28)$ \\
\hline Nausea/vomiting & $16(13.9)$ & $6(15)$ & $10(13.3)$ \\
\hline Diarrhoea & $19(16.5)$ & $7(17.5)$ & $12(16)$ \\
\hline Abdominal pain & $14(12.2)$ & $5(12.5)$ & $9(12)$ \\
\hline One of diarrhoea/nausea/abdominal pain & $35(30.4)$ & $17(42.5)$ & $18(24)^{*}$ \\
\hline Confusion & $20(17.4)$ & $3(7.5)$ & $17(22.7)^{*}$ \\
\hline Drowsiness & $14(12.2)$ & $2(5)$ & $12(16)^{*}$ \\
\hline Fall & $22(19.1)$ & $6(15)$ & $16(21.3)$ \\
\hline \multicolumn{4}{|l|}{ Presenting signs } \\
\hline High fever $\geq 38.0^{\circ} \mathrm{C}$ & $36(31.3)$ & $15(37.5)$ & $21(28)$ \\
\hline Low grade fever $37.5-37.9^{\circ} \mathrm{C}$ & $11(9.6)$ & $6(15)$ & $5(6.7)$ \\
\hline Normal temperature $36.0-37.4^{\circ} \mathrm{C}$ & $52(45.2)$ & $16(40)$ & $36(48)$ \\
\hline Low temperature $\leq 35.5^{\circ} \mathrm{C}$ & $12(10.4)$ & $1(2.5)$ & $11(14.7)^{*}$ \\
\hline Average oxygen saturations & $92 \%$ & $89 \%$ & $93 \%$ \\
\hline \multicolumn{4}{|l|}{ During hospital stay } \\
\hline$\geq 38.0^{\circ} \mathrm{C}$ (high-grade fever) & $61(53.0)$ & $26(65)$ & $35(46.7)$ \\
\hline$\geq 37.5^{\circ} \mathrm{C}$ (fever) & $79(68.7)$ & $31(77.5)$ & $48(64)$ \\
\hline $36.0-37.4^{\circ} \mathrm{C}$ (normal) & $25(21.7)$ & $3(7.5)$ & $22(29.3)^{*}$ \\
\hline$\leq 35.5^{\circ} \mathrm{C}$ (low temperature) & $54(47.0)$ & $7(17.5)$ & $47(62.7)^{*}$ \\
\hline$\leq 35.5^{\circ} \mathrm{C}$ and never $\geq 38.0^{\circ} \mathrm{C}$ & $24(20.9)$ & $4(10)$ & $20(26.7)^{*}$ \\
\hline
\end{tabular}

Values are numbers (percentages) unless stated otherwise. ${ }^{*} \mathrm{p}<0.05$, comparing the $<70$ and $\geq 70$ groups.

although there were no significant differences between the two groups. The relevant point is that a normal chest $X$-ray (around 1 in 3 overall), or one showing unilateral changes only (around one in five overall), does not exclude COVID-19. This reinforces the data relating to clinical presentation, emphasising that COVID-19 is more than a respiratory illness, with a much broader spectrum of disease.

\section{Clinical outcomes}

Mortality (22-25\%) was similar in the two groups (Table 4), likely representing the overall age and frailty of this late lockdown cohort, with a fifth of the younger cohort being admitted from a care home. Outcomes are known for all 115 patients.

\section{Previous tests}

$12.2 \%$ had had a previous negative swab result within the last 7 days, which represents a considerable underestimate of potential false negatives due to selection of the cohort according to positive swab result only. This adds to the overall literature reporting false negatives of up to $30 \%{ }^{2}$

\section{Discussion}

Many countries have criteria for access to testing for COVID-19, which is related to limitations of testing capacity. In the UK, community testing is reliant on the development of a cough or high fever, and more recently loss of taste or smell, said to be the 

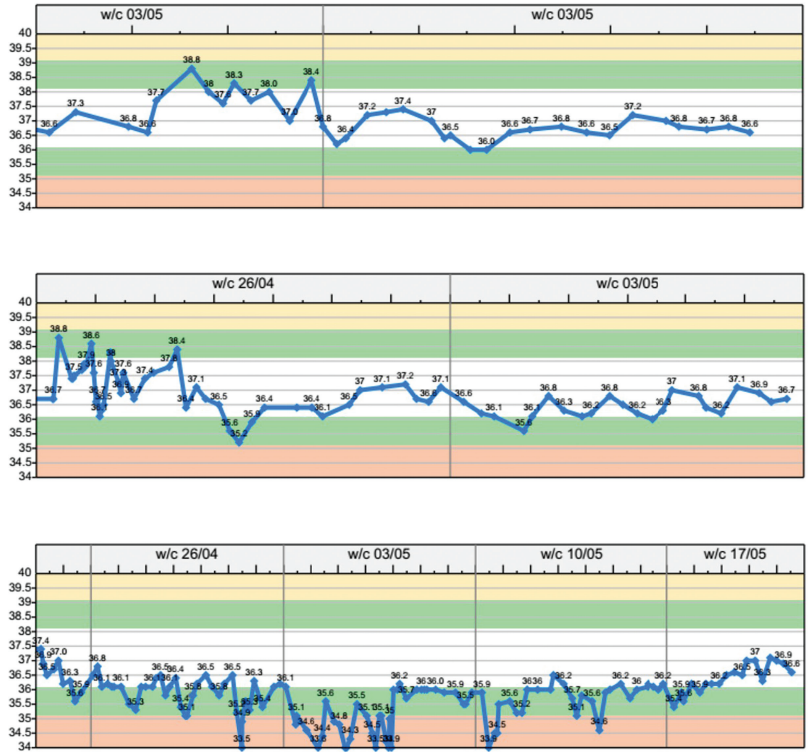

Fig 2. Example temperature traces, showing three patients with eithe high temperature spikes, high and low temperature spikes or only low temperature spikes.

cardinal signs of COVID-19. These data suggest that sticking to those criteria will disadvantage older people, whether living in their own homes or in care homes, as well as leading to potential misidentification of infectious patients presenting to hospital, increasing the risks of nosocomial infections. This is incredibly important as hospitals look to establish safe (often termed green) zones in which to restart elective surgery, cancer treatment and other essential services.

Our data on the range of presenting symptoms has some helpful similarities to that reported from the COVID Symptom Study smartphone-based app, ${ }^{7}$ going well beyond cough or high fever, although the presence or absence of loss of smell or taste, or hoarse voice, was not specifically commented on in the records for the cohort presented here. The presence of lethargy, loss of appetite, diarrhoea, delirium, abdominal pain and chest pain were frequently observed, but we would in particular draw attention to presentations with drowsiness, confusion and falls in the elderly. The two most common reasons for those over 70 presenting to hospital with COVID-19 were shortness of breath or falls.

Falls often lead to injuries, including head lacerations, skull fractures, subdural haematomas and fractured neck of femur. It is important for clinicians in the emergency, medical and surgical departments to be aware of this, as these prominent injuries can detract attention from the potential underlying cause for the fall, possibly contributing to nosocomial spread.

It is also interesting to note that presentations or clinical course involving low (rather than high) temperatures were more likely to occur in the elderly (Table 2 and Fig 2). Low or normal temperatures are a common feature of sepsis in the elderly, ${ }^{8}$ so it should not be a particular surprise to find this in association with COVID-19, but this has not been widely recognised. These temperature traces also illustrate why a one-off temperature screening measurement is not a sensitive test for COVID-19 given that many patients have a normal temperature much of the time.

Factors that may contribute to the different presentations of COVID-19 in the elderly may include immunosenescence, comorbidities and malnutrition, all of which have been associated with increased risk of infection and worse outcomes from sepsis in older adults. ${ }^{8}$ Body mass index was not recorded in this cohort, but there were significant differences in comorbidities and some laboratory parameters, lending further support to this.

\section{Strengths and limitations of this study}

This is a small but complete cohort, allowing a detailed analysis of all the patients diagnosed with COVID-19 in one hospital during a 2-week period late in lockdown. It includes patients across the spectrum of disease severity, on wards as well as the intensive care unit. It therefore has the strengths of considerable depth of information, and represents an entire cohort according to positive RT-PCR swab test, presenting to one representative district general
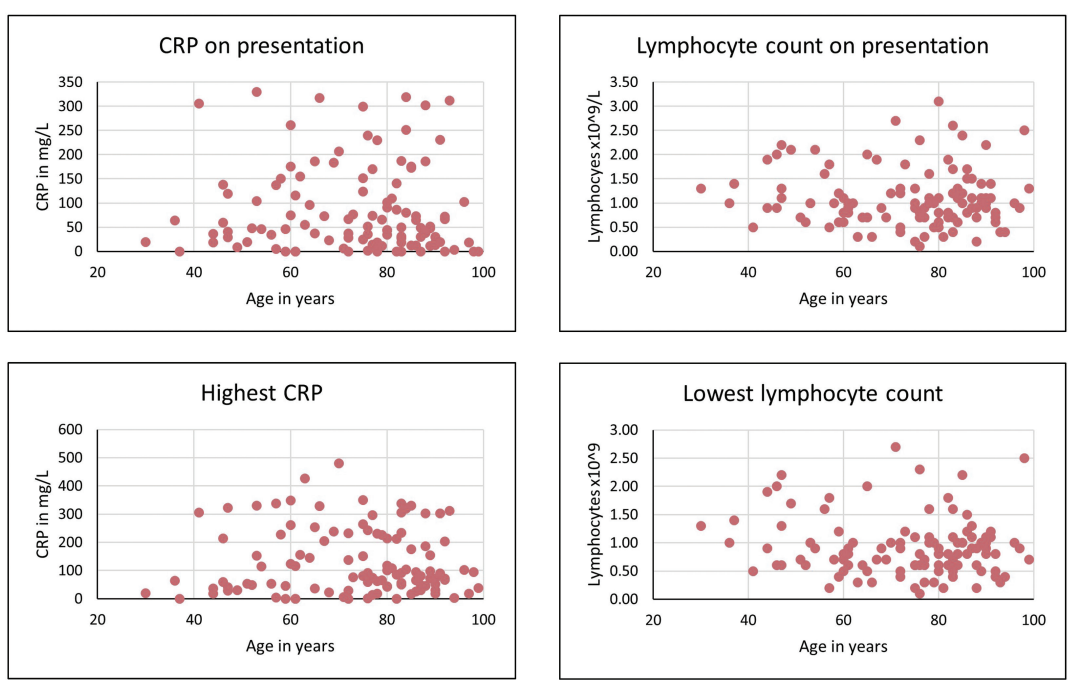
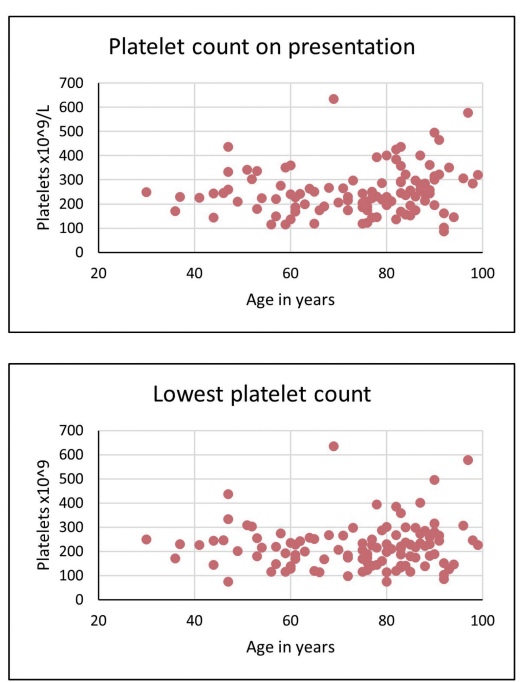

Fig 3. Key laboratory parameters across the age range. 
Table 3. Laboratory investigations at diagnosis and during admission

\section{Laboratory investigation}

Lymphocyte count on presentation $\times 10^{9} / \mathrm{L}$ (NR 1.4-4.8)

Lowest lymphocyte count $\times 10^{9} / \mathrm{L}$

Platelet count on presentation $\times 10^{9} / \mathrm{L}$

(NR 150-400)

Lowest platelet count $\times 10^{9} / \mathrm{L}$

White cell count on presentation $\times 10^{9} / \mathrm{L}$

(NR 4.0-11.0)

Highest white cell count $\times 10^{9} / \mathrm{L}$

CRP on presentation $\mathrm{mg} / \mathrm{L}(\mathrm{NR}<5)$

Highest CRP mg/L

\section{Under 70 survivors}

1.0

$(0.73-1.3)$

0.9

$(0.6-1.3)$

244.5

(180.75-273)

235.5

(173-256.5)

6.25

(5.28-8.93)

7.05

(5.6-12.3)

57.0

(30.25-133.25)

61.5

(37.25-223.75)
Under 70 died

1.0

(0.58-1.93)

0.7

(0.5-0.9)

225.5

(181-231.25)

191

(145.5 - 226.5)

8.9

(7.0-12.1)

$13.15^{*}$

(11.18-17.53)

$126.5^{*}$

(66.25-196.5)

$232.5^{*}$

(115.5-314.0)
Over 70 survivors

0.95

(0.7-1.3)

0.8

(0.6-1.0)

225.0

(180.5-288.5)

197.0

(147.5-256)

8.7

(6.7-12.35)

9.85

(7.0-14.38)

58.0

(25.75-101.75)

80

(50.0-214.0)
Over 70 died

1.0

(0.78-1.33)

0.9

(0.63-1.13)

289*

(242.5-395.75)

236.5

(187-273)

$13.1^{*}$

(9.1-15.65)

$14.05^{*}$

(13.18-19.55)

49.0

(30-207)

96

(54.25-217.5)

Values are median (interquartile range). $C R P=C$-reactive protein; $N R=$ normal range. ${ }^{*} \mathrm{p}<0.05$, comparing those who survived to those who died within each age-group cohort.

hospital in the UK, with a catchment area of around 500,000 people. This degree of detailed analysis complements larger scale studies that are unable to interrogate clinical records to this level of detail.

Those who may have had COVID-19 but had a single negative swab could not be identified for inclusion in the study. The observation that 1 in 8 detected cases had had a previous negative test suggests that there were more cases to be identified. The

\begin{tabular}{|c|c|c|c|}
\hline Characteristic & $\begin{array}{l}\text { Whole } \\
\text { cohort }\end{array}$ & $\begin{array}{l}\text { Under } \\
70\end{array}$ & $\begin{array}{l}\text { Aged } \\
\geq 70\end{array}$ \\
\hline \multicolumn{4}{|c|}{ CXR (9 did not have a CXR) } \\
\hline No consolidation & $37(34.9)$ & $12(31.6)$ & $25(36.7)$ \\
\hline Unilateral consolidation & $23(21.7)$ & $6(15.7)$ & $17(25.0)$ \\
\hline Bilateral consolidation & $43(40.5)$ & $20(52.6)$ & $23(33.8)$ \\
\hline \multicolumn{4}{|l|}{ Outcome } \\
\hline Discharged & $87(75.7)$ & $31(77.5)$ & $56(74.7)$ \\
\hline Died & $28(24.3)$ & 9 (22.5) & $19(25.3)$ \\
\hline Died 30-39 $(n=3)$ & & 0 & \\
\hline Died 40-49 $(n=9)$ & & $1(11.1)$ & \\
\hline Died 50-59 $(n=13)$ & & $3(23.1)$ & \\
\hline Died 60-69 $(n=15)$ & & $5(33.3)$ & \\
\hline Died 70-79 $(n=22)$ & & & $2(9.1)$ \\
\hline Died 80-89 $(n=35)$ & & & $9(25.7)$ \\
\hline Died 90-99 $(n=18)$ & & & $8(44.4)$ \\
\hline
\end{tabular}

Values are numbers (percentages). $\mathrm{n}$ represents the total number of patients in each age group. $C X R=$ chest radiograph. question of how to interpret a coronavirus test result is also essential to planning of bio-safe zones in hospital, and the principles were recently clearly explained. ${ }^{2}$ Interpreting the test depends on the pre-test probability, and the data presented here should contribute to a greater awareness of the potential clinical presentations with COVID-19 in the elderly, allowing pre-test probability to be adjusted accordingly.

The study contains sufficient detail to gain insight into the likely source of infections and range in clinical presentations in older patients presenting with COVID-19. Determination of source of infection would require resources for detailed contact tracing and genetic analysis of the viral genome. For example, several patients lived in care homes but had also had recent admissions to hospital, with a potential incubation period of 14 days making it difficult to identify the source of infection within the heath and care sector. The cohort is not powered to look at mortality rates at scale, but this information has been provided due to interest of colleagues and patients.

Validation of the reason for presentation of the third of elderly patients who have neither cough nor fever will require a larger cohort, which undoubtedly now exist at a national level in several countries, and we hope that our work demonstrates that this deserves urgent attention. Perhaps it may be a subject for further consideration by the ISARIC $4 C$ investigators. ${ }^{6}$

\section{Conclusions}

The vast majority of cases of COVID-19 in weeks 5 and 6 of lockdown arose in those in contact with health and care services, with 2 in 5 cases unequivocally arising in the health and care environment as they were in care home residents. This is a critical public health consideration for second wave planning and for countries still in the early phase of their epidemic. Carers visiting people in their own homes may also potentially carry coronavirus-19, and while considerable attention has been paid to testing and 
protective equipment in care homes and hospitals, carers in the broader sense need to be similarly protected and thought about. COVID-19, like many infectious diseases, can present differently in the elderly, and is more than simply a respiratory disease. Therefore focusing testing on those with a cough or fever will miss at least 1 in 3 cases in those over the age of 70 . Presentations overlap with many medical conditions, and swab tests are not perfect, so it is likely that a strategy encompassing a high degree of suspicion, taking into account epidemiological risk factors, alongside the availability of repeated and regular testing of patients and staff will be needed to limit nosocomial spread in hospitals. ${ }^{11}$

\section{References}

1 Onder G, Rezza G, Brusaferro S. Case-fatality rate and characteristics of patients dying in relation to COVID-19 in Italy. JAMA 2020;323:1775-6.

2 Watson J, Whiting PF, Brush JE. Interpreting a COVID-19 test result. BMJ 2020;369:m1808.

3 Guan WJ, Ni ZY, Hu Y et al. Clinical characteristics of coronavirus disease 2019 in China. N Engl ] Med 2020;382:1708-20.

4 Inciardi RM, Adamo M, Lupi L et al. Characteristics and outcomes of patients hospitalized for COVID-19 and cardiac disease in Northern Italy. Eur Heart ] 2020;1821-9.

5 Goyal P, Choi J], Pinheiro LC et al. Clinical characteristics of COVID-19 in New York City. N Engl J Med 2020;382:2372-4.
6 Docherty AB, Harrison EM, Green CA et al. Features of 20133 UK patients in hospital with COVID-19 using the ISARIC WHO Clinical Characterisation Protocol: prospective observational cohort study. BMJ 2020;369:m1985.

7 Menni C, Valdes AM, Freidin MB et al. Real-time tracking of self-reported symptoms to predict potential COVID-19. Nat Med 2020;26:1037-40.

8 Clifford KM, Dy-Boarman EA, Haase KK et al. Challenges with diag nosing and managing sepsis in older adults. Expert Rev Anti Infect Ther 2016;14:231-41.

9 Zhou F, Yu T, Du R et al. Clinical course and risk factors for mortality of adult inpatients with COVID-19 in Wuhan, China: a retrospective cohort study. Lancet 2020;395:1054-62.

10 Ruan Q, Yang K, Wang W, Jiang L, Song J. Clinical predictors of mortality due to COVID-19 based on an analysis of data of 150 patients from Wuhan, China. Intensive Care Med 2020;46:846-8.

11 Rivett L, Sridhar S, Sparkes D et al. Screening of healthcare workers for SARS-CoV-2 highlights the role of asymptomatic carriage in COVID-19 transmission. ELife 2020;9:e58728.

Address for correspondence: Dr Sybil Stacpoole, North West Anglia NHS Foundation Trust (NWAFT), Peterborough City Hospital, Bretton Gate, Bretton, Peterborough, Cambridgeshire PE3 9GZ.

Email: sybilstacpoole@nhs.net 